\title{
Capacidad de Disolución del Hipoclorito de Sodio con o sin Activación
}

\section{Dissolution Capacity of Sodium Hypochlorite with or without Activation}

\author{
H. Monardes Cortés*, MT. Martínez Uribe*, F. Arriagada Arriagada*, J. Abarca Reveco.*
}

\begin{abstract}
RESUMEN
Introducción. Una de las propiedades del hipoclorito de sodio $(\mathrm{NaOCl})$ es la disolución de tejido pulpar remanente después de la instrumentación. Los sistemas de activación del irrigante pretenden mejorar esta propiedad. Este trabajo tiene como objetivo determinar la capacidad de disolución de tejido de $\mathrm{NaOCl}$ con y sin activación sónica o ultrasónica en diferentes concentraciones.

Metodología. 300 muestras de tejido conectivo de paladar de cerdo de 4,5* $2 \mathrm{~mm}$ obtenidos 1 día antes del estudio, congelado a $-15^{\circ} \mathrm{C}$ en $100 \%$ de humedad, fueron pesados en una balanza analítica y sometidos a la acción de $\mathrm{NaOCl} 1 \%, 3 \%$ y 5\% con y sin activación durante 45 minutos, cambiando la solución cada 10 minutos. Se secaron en papel filtro y se pesaron nuevamente. Los datos se analizaron mediante tests Kolmogorov - Smirnov; Kruskal - Wallis y Mann - Whitney.

Resultados y Discusión. NaOCl 1\% tiene menor capacidad de disolución que mejora levemente al activarlo. $\mathrm{NaOCl} 3 \%$ tiene mejor capacidad de disolución que $\mathrm{NaOCl} 1 \%$, pero la activación no la mejora significativamente. $\mathrm{NaOCl} 5 \%$ tiene mayor capacidad de disolución, que aumenta con la activación, sin significancia entre activación sónica y ultrasónica, lo que se debería a que la mucosa palatina porcina requiere más tiempo para su disolución completa, y a que el volumen de tejido utilizado es mayor que una pulpa dental.

Conclusiones. Bajo las condiciones de este estudio, sólo $\mathrm{NaOCl} 5 \%$ mostró mayor capacidad de disolución con la activación. $\mathrm{NaOCl} 1 \%$ y 3\% no mejoraron significativamente con la activación.
\end{abstract}

PALABRAS CLAVE: Hipoclorito de sodio, disolución de tejidos, activación.

\begin{abstract}
Introduction. One of the properties of sodium hypochlorite $(\mathrm{NaOCl})$ is dissolution of pulp tissue remaining after instrumentation. Irrigant activation systems aim to improve this property. The objective of this work is to determine tissue dissolution capacity of $\mathrm{NaOCl}$ with and without sonic or ultrasonic activation in different concentrations.

Methodology. 300 pork palate connective tissue samples of $4.5 * 2 \mathrm{~mm}$ obtained 1 day before the study, frozen at $-15^{\circ} \mathrm{C}$ in $100 \%$ humidity, weighed on an analytical balance and subjected to the action of $\mathrm{NaOCl}$ $1 \%, 3 \%$ and $5 \%$ with and without activation for 45 minutes, changing the solution every 10 minutes. They were dried on filter paper and weighed again. The data were analyzed by Kolmogorov-Smirnov tests; Kruskal - Wallis and Mann - Whitney.

Results and Discussion. $\mathrm{NaOCl} 1 \%$ has a lower dissolution capacity that improves slightly when activated.

* Programa de Especialización en Endodoncia, Facultad de Odontología, Universidad San Sebastián, Santiago, Chile.
\end{abstract}


$\mathrm{NaOCl} 3 \%$ has better dissolution capacity than $\mathrm{NaOCl} 1 \%$, but the activation does not significantly improve it. $\mathrm{NaOCl} 5 \%$ has greater dissolution capacity, which increases with activation, without significance between sonic and ultrasonic activation, which is due to the fact that the porcine palatine mucosa requires more time for its complete dissolution, since the volume of tissue used is greater than a dental pulp Conclusions. Under the conditions of this study, only $\mathrm{NaOCl} 5 \%$ showed greater dissolution capacity with activation. $\mathrm{NaOCl} 1 \%$ and $3 \%$ did not improve significantly with activation.

KEY WORDS: Sodium hypochlorite, tissue dissolution, activation.

Fecha de recepción: 27 de junio de 2019

Fecha de aceptación: 9 de julio de 2019.

H. Monardes Cortés, MT. Martínez Uribe, F. Arriagada Arriagada, J. Abarca Reveco.

Capacidad de Disolución del Hipoclorito de Sodio con o sin Activación.

2019; 35, (3): 114-119.

\section{INTRODUCCIÓN}

La eliminación de restos pulpares vitales y necróticos, microorganismos y toxinas microbianas de los canales radiculares, es esencial para el éxito del tratamiento en endodoncia.

Durante la preparación químico - mecánica de los canales radiculares, los instrumentos eliminan parte de los contenidos del canal, la irrigación juega un papel indispensable en la eliminación de tejido orgánico1, en particular, en aquellas partes que son inaccesibles para la instrumentación por la compleja anatomía radicular ${ }^{2}$.

El hipoclorito de sodio ( $\mathrm{NaOCl}$ ) es el irrigante del canal radicular más comúnmente utilizado, entre sus características destacan propiedades antimicrobianas y la capacidad de disolución de tejidos orgánicos $^{2}$. Es por esto, que se han descrito e investigado diversas maneras de mejorar la eficacia de las preparaciones de $\mathrm{NaOCl}$ en la disolución del tejido, entre otras, aumentando la concentración, el $\mathrm{pH}^{3}$ y la temperatura de las soluciones, incorporando la activación sónica o ultrasónica ${ }^{2}$.

El objetivo de la presente investigación es determinar qué tan efectiva es la concentración y activación de $\mathrm{NaOCl}$ en la disolución de tejido orgánico.

\section{MATERIALES Y MÉTODOS}

Se realizó un estudio experimental in vitro, utili-

zando $\mathrm{NaOCl}$ en 3 diferentes concentraciones, $1 \%$, 3\% y 5\% (Laboratorio Hertz, Chile), de fabricación reciente y $\mathrm{pH}$ promedio 11.5 , los cuales fueron mantenidos antes y durante su uso, en un contenedor frío, oscuro y ventilado.

Como tejido de estudio se utilizó tejido conectivo de paladar de cerdo recién faenado con fines de alimentación humana, en un recinto debidamente autorizado por la autoridad sanitaria, el cual fue obtenido un día antes del estudio experimental y mantenido congelado a $-15{ }^{\circ} \mathrm{C}$ con $100 \%$ de humedad, hasta 30 minutos antes del inicio del trabajo experimental. El tejido fue cortado en piezas de 4,5 x $2 \mathrm{~mm}$ con un bisturí circular (Biohorizons Tissue Puch $4.5 \mathrm{~mm}$, Alabama, USA) diseñado para la obtención de este tipo de muestras, puesto

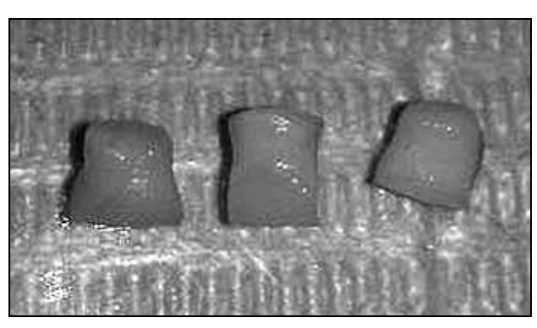

Figura 1. Muestras de tejido conectivo de paladar de cerdo de $4,5 \mathrm{~mm} \times 4,5 \mathrm{~mm}$. que el área de superficie tiene un impacto importante en la disolución de tejido, por lo que cada muestra debe tener una forma $y$ tamaño similar (Figura 1).

Las muestras fueron pesadas previamente en una balanza analítica (Adam Equipment, ACBplus-600H. Milton Keynes, U.K.) y distribuidas aleatoriamente en 1 grupo control y 9 grupos experimentales de 30 muestras cada uno: 
-Grupo 1: Tejido en cloruro de sodio al 0,9\%

-Grupo 2: Tejido en $\mathrm{NaOCl}$ al 1\%, no activado

-Grupo 3: Tejido en $\mathrm{NaOCl}$ al 1\%, activación sónica

-Grupo 4: Tejido en $\mathrm{NaOCl}$ al 1\%, activación ultrasónica

-Grupo 5: Tejido en $\mathrm{NaOCl}$ al 3\%, no activado

-Grupo 6: Tejido en $\mathrm{NaOCl}$ al 3\%, activación sónica

-Grupo 7: Tejido en $\mathrm{NaOCl}$ al 3\%, activación ultrasónica

-Grupo 8: Tejido en $\mathrm{NaOCl}$ al 5\%, no activado

-Grupo 9: Tejido en $\mathrm{NaOCl}$ al 5\%, activación sónica

-Grupo 10: Tejido en $\mathrm{NaOCl}$ al 5\%, activación ultrasónica.

Las muestras de los grupos 2, 5 y 8 fueron depositadas en un frasco de vidrio de $7 \mathrm{ml}$, el cual contenía $\mathrm{NaOCl}$ al $1 \%, \mathrm{NaOCl}$ al 3\% e $\mathrm{NaOCl}$ al $5 \%$ respectivamente, por un tiempo de $45 \mathrm{mi}-$ nutos, realizando recambio total de la solución cada 10 minutos. Luego, las muestras se dejaron en papel filtro por 5 minutos para absorber la solución residual. Finalmente, se procedió a hacer el pesaje final.

Las muestras de los grupos 3, 6 y 9 fueron sometidas al mismo procedimiento, pero además se realizaron 3 ciclos de activación de la solución por un período de 30 segundos, con un sistema de vibración sónica Endoactivator ${ }^{\circledR}$ (Denstply, Maillefer, Suiza), con una punta de polímero flexible de tamaño 25/0.4, la cual fue accionada en el frasco, sin tocar las muestras.

Las muestras de los grupos 4,7 y 7 fueron sometidas al mismo procedimiento anterior, pero se activaron con un sistema de vibración ultrasónica compuesto por un ultrasonido Suprasson P5 Newtron ${ }^{\circledR}$ (Satelec Acteon, Merignac Cedex, France) y una punta ultrasónica VDW Irri K (VDW GmbH, Munich, Germany).

Los datos obtenidos se registraron en una planilla Excel especialmente diseñada y sometidas a análisis estadístico utilizando el software estadístico Stata V 15.1.

\section{RESULTADOS}

Para determinar la distribución de la muestra se aplicó el Test de Kolmogorov-Smirnov (Statistic

\begin{tabular}{llllll}
\multicolumn{5}{c}{ Tabla } & 1. Pérdida de \\
Activación & Grupo & Media $\%$ & Máx & Min & Ranking \\
Sin Activación & $2(1 \%)$ & 50.34 & 70.00 & 25.00 & 45.00 \\
& $5(3 \%)$ & 80.74 & 90.91 & 62.50 & 28.41 \\
& $8(5 \%)$ & 79.61 & 90.91 & 70.00 & 20.91 \\
Activación & $3(1 \%)$ & 47.50 & 58.30 & 13.22 & 45.08 \\
Sónica & $6(3 \%)$ & 80.94 & 92.80 & 50.25 & 42.55 \\
& $9(5 \%)$ & 87.65 & 98.82 & 70.91 & 27.91 \\
Activación & $4(1 \%)$ & 43.87 & 77.09 & 19.50 & 57.59 \\
Ultrasónica & $7(3 \%)$ & 78.03 & 87.00 & 46.25 & 40.75 \\
& $1(5 \%)$ & 85.36 & 97.50 & 74.44 & 23.06 \\
\hline Total & & 70.45 & 98.82 & 13.22 & 85.60 \\
\hline
\end{tabular}

\begin{tabular}{lc}
\multicolumn{3}{c}{ Tabla 2. Prueba Kruskall-Wallis } \\
& DP\% \\
Chi-Square & 186,71 \\
df & 8 \\
Sig. & 000 \\
\hline
\end{tabular}

$=0.152 ; \mathrm{p}<0,001)$ y se determinó que tenía una distribución no paramétrica. Posteriormente se aplicaron las pruebas de Kruskal-Wallis $(p<0,05)$ (Tabla 2) y de Mann-Whitney $(p<0,05)$ (Tabla 3), encontrándose diferencias significativas entre grupos.

$\mathrm{NaOCl}$ al $1 \%$ tiene un menor efecto en la disolución del tejido orgánico, mejorando levemente sus propiedades de disolución al aplicar activación ultrasónica $(\mathrm{p}=0.018)$. Para $\mathrm{NaOCl}$ al 3\%, ni la activación sónica $(p=0.734)$ ni la activación ultra-

\begin{tabular}{|c|c|c|c|c|c|c|c|c|}
\hline & 3\% Sin Activación & $5 \%$ Sin Activación & 1\% Sónica & 3\% Sónica & 5\% Sónica & 1\% Ultrasónica & 3\% Ultrasónica & 5\% Ultrasónica \\
\hline 1\% Sin Activación & $0.000^{*}$ & $0.000 *$ & 0.605 & $0.000 *$ & $0.000 *$ & $0.018^{*}$ & $0.000 *$ & $0.000 *$ \\
\hline 3\% Sin Activación & & 0.434 & $0.000 *$ & 0.734 & $0.003 *$ & $0.000 *$ & 0.141 & 0.336 \\
\hline 5\% Sin Activación & & & $0.000 *$ & 0.260 & $0.000 *$ & $0.000 *$ & 0.970 & $0.003^{*}$ \\
\hline 1\% Sónica & & & & $0.000 *$ & $0.000 *$ & $0.037 *$ & $0.000 *$ & $0.000 *$ \\
\hline 3\% Sónica & & & & & $0.009 *$ & $0.000^{*}$ & 0.062 & $0.030 *$ \\
\hline 5\% Sónica & & & & & & $0.000 *$ & $0.000 *$ & 0.311 \\
\hline
\end{tabular}

* Significativo al $5 \%(a=0.05)$ 
sónica $(p=0.141)$ mejoran significativamente las propiedades de disolución, pero se observa una mayor capacidad de disolución de tejido orgánico con respecto a la concentración del 1\% ( $\mathrm{p}=0.000)$.

La activación sónica $(\mathrm{p}=0.000)$ y ultrasónica $(p=0.003)$ aumentan las propiedades de disolución de $\mathrm{NaOCl}$ al 5\%, sin embargo, no se observan diferencias significativas, entre la activación sónica y ultrasónica $(\mathrm{p}=0.311)$.

La mayor disolución de tejido se observa en $\mathrm{NaOCl}$ al $5 \%$, comparado con $1 \%$ y $3 \%(p=0.000)$, siendo mejorada su capacidad de disolución cuando se realiza activación.

\section{DISCUSIÓN}

$\mathrm{NaOCl}$ ha demostrado tener capacidad de disolución de materia orgánica, lo que depende de varios factores: concentración, temperatura, condiciones de almacenamiento ${ }^{4}, \mathrm{Ph}^{5}$ cantidad de materia orgánica y de tejido disponible ${ }^{6}$, y de la activación de la irrigación 7 . Además, se ha reportado una acción solvente sobre las células muertas, que acelera la separación del tejido muerto del tejido vivo ${ }^{8}$.

Ramírez - Bower ${ }^{9}$ comprobó que la pérdida de materia orgánica de la dentina - colágeno expuesto a $\mathrm{NaOCl} 2,5 \%$ - es de $40 \%$ a los $2 \mathrm{~min}$, $60 \%$ entre los 6 y los $10 \mathrm{~min}$, pues el tiempo de saturación del $\mathrm{NaOCl}$ comienza a los 5 minutos de entrar en contacto con materia orgánica, por lo tanto, es necesario el recambio de la solución, ya que a menor disponibilidad de iones hidroxilo menor capacidad de disolución, lo que también sucede con el polvo de dentina ${ }^{10}$.

$\mathrm{NaOCl}$ se inactiva al interactuar con materia orgánica ${ }^{11}$, debido a que se producen reacciones de saponificación, neutralización, y formación de cloraminas, reacciones que ocurren principalmente en la superficie de contacto entre $\mathrm{NaOCl}$ y materia orgánica, llevan a la licuefacción del tejido $^{11}$ y las moléculas de $\mathrm{NaOCl}$ involucradas se consumen, resultando en una disminución de su concentración y actividad local. Además, soluciones acuosas de $\mathrm{NaOCl}$ tienen altos valores de tensión superficial, lo que disminuye su capacidad de humectación, por lo que la adición de surfactantes mejoraría esta propiedad ${ }^{12}$.
La mucosa palatina porcina se ha usado para evaluar la capacidad de disolución de los tejidos de las soluciones de irrigación ${ }^{13}$. Cuando se compara con el tejido de la pulpa humana, se ha encontrado que la mucosa palatina porcina requiere un tiempo más largo para su disolución completa, sin embargo, no se han encontrado diferencias en términos de la capacidad de solubilización ${ }^{13}$.

Teniendo en cuenta que la cantidad de materia orgánica y la superficie disponible influyen en el poder de disolución de $\mathrm{NaOCl}^{14}$, vale la pena señalar que el volumen del tejido utilizado en este estudio es más grande que en la realidad.

$\mathrm{Al}$ igual que en este estudio, los modelos in vitro a menudo no replican la realidad clínica ${ }^{15}$ pero, permiten saber qué tan efectiva es la activación para acelerar los procesos de disolución de tejido, sin considerar otros factores clínicos. Se ha reportado que la activación / agitación del irrigante mejora de manera importante la acción antibacteriana y la acción de disolución de tejidos del $\mathrm{NaOCl}^{16}$.

En el presente estudio, se realizaron 3 ciclos de activación de $30 \mathrm{seg}$, ya que diversos estudios habían demostrado que el uso de activación de 30 seg es el más efectivo ${ }^{17}$ y que según Estévez ${ }^{18}$, tiempos mayores a $30 \mathrm{seg}$ pueden producir un "efecto meseta" donde la agitación se estabiliza. Estudios similares han considerado $30 \mathrm{seg}^{19}$ y 2 ciclos de $30 \mathrm{seg}^{20}$.

Estudios ex vivo en canales radiculares con flujo restringido a través del foramen apical, que simula mejor el flujo de fluidos en condiciones clínicas, revelaron que la penetración de soluciones se mejora con ultrasonido y, en menor grado con activación sónica ${ }^{19}$. Sin embargo, en el presente estudio, sólo para $\mathrm{NaOCl} 5 \%$ se pudo observar una mayor disolución de tejido al realizar activación. Para las concentraciones 1\% y 3\%, la activación no mejoró la disolución de tejido.

Tal como lo demuestran estudios anteriores, el tiempo de degradación del tejido por la acción de $\mathrm{NaOCl}$ es directamente proporcional a la concentración, más que a la activación. Sin embargo, los sistemas de activación mejorarían su capacidad de disolución sólo en la práctica clínica y no en estudios in vitro, lo se explicaría por la capacidad de $\mathrm{NaOCl}$ de poder difundir a 
la complejidad anatómica propia de los canales radiculares ${ }^{21}$. Asimismo, la adición de otros elementos surfactantes y ácido glicólico, tendría una acción sinérgica mejorando la disolución de tejidos $^{22}$.

\section{BIBLIOGRAFIA}

1. Hasse MGM, Gonçalves JF, Spano JCE, Silveira LFM, Martos J, Barbin EL. Solvent effect of Sodium hypochlorite solutions commercialized in Pelotas (Brazil) on bovine dental pulps. Dental Press Endod. 2014; 42(2): 45-51

2. Haapasalo M, Shen Y, Wang Z, Gao Y. Irrigation in endodontics. Br Dent J. 2014; 216(6): 299-03

3. Van der Waal S, Connert T, Laheij A, de Soet J, Wesselink P. Free available chlorine concentration in sodium hypochlorite solutions obtained from dental practices and intended for endodontic irrigation: Are the expectations true? Quintessence Int. 2014; 45(6): 467 - 74

4. Plotino G, Cortese T, Grande NM, Leonardi DP, Di Giorgio G, Testarelli L, Gambarini G. New technologies to improve root canal disinfection. Braz Dent J. 2016; 27(1): 3 -8

5. Del Carpio-Perochena A, Monteiro Bramante C, Bombarda de Andrade F, Alves Maliza AG, Cavalini Cavenago B, Marciano MA, Amoroso-Silva $P$, Hungaro Duarte $M$. Antibacterial and dissolution ability of Sodium hypochlorite in different $\mathrm{pHs}$ on multi-species biofilms. Clin Oral Invest. 2015; 19: 2067- 73

6. Quintana RM. Jardine AP, Montagner F, Fatturi Parolo CC, Morgental RD, Poli Kopper PM. Effect of human, dentin, albumin and lypopolisaccharide on the antibacterial activity of endodontic activity of endodontic irrigants. J Conserv Dent. 2017; 20: 341 - 45

7. Tejada S, Baca P, Ferrer-Luque CM, Ruiz-Linares $M$, Valderrama MJ, Arias-Moliz MT. Influence of dentine debris and organic tissue on the properties of sodium hypochlorite solutions. Int Endod J. 2019; 52(1): 114 - 22

8. Keyes M, Thibodeau R. Dakin solution. (So- dium Hypochlorite). StatPearls. 2018; Oct 27

9. Ramírez-Bommer C, Gulabivala K, Ng Y-L, Young A. Estimated depth of apatite and collagen degradation in human dentine by sequential exposure to sodium hypochlorite end EDTA: quantitative FTIR study. Int Endod J. 2018; 51(4): 469 - 78

10. Morgental RD, Singh A, Sappal H, Kopper PMP, Vier-Pelisser FV, Peters OA. Dentin inhibits the antibacterial effects of new and conventional endodontic irrigants. JEndod. 2013; 39(3): $406-10$

11. Topbas C, Adiguzel O. Endodontic irrigation solutions. A review. Int Dent Res. 2017; 7: 54 $-61$

12. Iglesias JE, Pinheiro LS, Weibel DE, Montagner $F$, Greca FS. Influence of surfactans addition on the properties of calcium hypochlorite solutions. J Appl Oral Sci. 2019; 27: e20180157

13. Naenni N, Thoma K, Zehnder M. Soft tissue dissolution capacity of currently used and potential endodontic irrigants. J Endod. 2004; 30(11): $785-87$

14. Moorer WR, Wesselink PR. Factors promoting the tissue dissolving capability of sodium hypochlorite. Int Endod J. 1982; 25(4): 187 - 96

15. Sobhani OE, Gulabivala K, Knowles JC, Ng YL. The effect of irrigation time, root morphology and dentin thickness on tooth surface strain when using $5 \%$ sodium hypochlorite and 17\% EDTA. Int Endod J. 2010; 43(3): 190-99

16. Landolo A, Amato M, Dagna A, Poggio C, Abellatif D, Franco V, Pantaleo G. Intracanal heating of sodium hypochlorite: Scanning electron microscope evaluation of root canal walls. J Conserv Dent. 2018; 21(5): 569- 73

17. Plotino G, Pameijer CH, Grande NM, Somma F. Ultrasonics in endodontics: a review of the literature. J Endod. 2007; 33(2): 81 - 95

18. Estevez R, Conde AJ, Valencia de Pablo O, de la Torre F, Rossi-Fedele G, Cisneros R. Effect of passive ultrasonic activation on organic Tissue dissolution from simulated grooves in root 
Canals using Sodium hypochlorite with or without surfactants and EDTA. J Endod. 2017; 43(7): 1161-65

19. Sáinz-Pardo M, Estevez R, Pablo ÓV, Rossi-Fedele G, Cisneros R. Root canal penetration of a Sodium hypochlorite mixture using Sonic or ultrasonic activation. Braz Dent J. 2014; 25(6): 489-93

20. Conde AJ, Estevez R, Loroño G, Valencia de Pablo O, Rossi-Fedele G, Cisneros R. Effect of Sonic and ultrasonic Activation on organic Tissue dissolution from simulated grooves in root Canals using Sodium hypochlorite and EDTA. In Endod J. 2017; 50(10): 976-82

21. Vera J, Arias A, Romero M. Dynamic movement of intracanal gas bubbles during cle- aning and shaping procedures: the effect of maintaining apical patency on their presence in the middle and cervical thirds of human root canals - an in vivo study. $J$ Endod. 2012; 38(2): 200-3

22. Peña Lopez A, Conde AJ, Estevez R, Valencia de Pablo O, Rossi-Fedele G, Cisneros F. Sodium hypochlorite and a preparation containing glycocholic acid and surfactans have a synergistic action on organic tissue dissolution In Vitro. J Endod. 2018; 44(5): 813 - 15

\section{AUTOR DE CORRESPONDENCIA:}

Dirección Postal:

Bellavista 7, piso 8

Recoleta, Santiago, Chile

e-mail: hector.monardes@uss.cl 